\title{
STICHWORT
}

\section{Selbstverletzendes Verhalten}

\author{
Klaus Sarimski
}

\begin{abstract}
Selbstverletzendes Verhalten bei Menschen mit intellektueller Beeinträchtigung ist ein gegen den eigenen Körper gerichtetes Verhalten, das wiederholt und in stereotyper Form auftritt und das direkte Gewebeschädigungen am Körper des Betroffenen verursacht (oder verursachen würde, sofern keine schützenden Maßnahmen ergriffen werden). In der ICD-10 und im DSM-V werden diese Verhaltensweisen jeweils den psychischen und Verhaltensstörungen zugeordnet und als "Stereotype Bewegungsstörung mit Selbstverletzung" (F98.41, bzw. 307.3) klassifiziert. Als diagnostische Merkmale werden in beiden Klassifikationssystemen ihr repetitiver, scheinbar getriebener, nicht funktionaler Charakter, die dadurch entstehenden körperlichen Verletzungen sowie die Beeinträchtigung von „normalen“ Aktivitäten hervorgehoben.
\end{abstract}

Die häufigsten selbstverletzenden Verhaltensweisen bei Kindern, Jugendlichen und Erwachsenen mit intellektueller Beeinträchtigung sind das Kopfschlagen, Beißen oder Schlagen des eigenen Körpers. Mit geringerer Häufigkeit tritt Pica (Schlucken von nicht essbaren Gegenständen), Ruminieren (Hochwürgen von Nahrung), Kratzen und Kneifen auf (Bienstein \& Rojahn 2013). Pica und Ruminieren werden dabei in den o.g. Klassifikationssystemen als eigenständige Störungsbilder behandelt. Die meisten betroffenen Personen entwickeln mehrere Symptome, die in Abhängigkeit des Situationskontextes variieren können, intraindividuell jedoch relativ stabil sind. Die Symptome treten in vielen Fällen bereits in der Kindheit auf und weisen dann eine hohe Persistenz auf. Intensität und Auftretenshäufigkeit sind sehr unterschiedlich.
Prävalenzschätzungen von selbstverletzendem Verhalten kommen aufgrund unterschiedlicher Definitionen, verwendeter Messinstrumente, Zielgruppen und Untersuchungsperioden zu recht weit variierenden Angaben. In einer populationsweiten Erhebung, in die alle Kinder, Jugendliche und Erwachsene in Kalifornien einbezogen waren, stellten Rojahn et al. (1993) bei $8.1 \%$ das aktuelle Vorliegen selbstverletzenden Verhaltens fest. In einer Erhebung bei Jugendlichen und Erwachsenen, die außerhalb von Behinderteneinrichtungen lebten, ermittelten Cooper et al. (2009) eine Prävalenz von 4.9\%. Generell variiert die Auftretensrate mit dem Alter, dem Schweregrad der Behinderung und dem Lebensumfeld der untersuchten Gruppe. Eine hochgradige geistige Behinderung, fehlende sprachliche Kommunikationsfähigkeiten und restriktivere Wohn- und Lebensbedingungen in Heimeinrichtungen begünstigen ihr Auftreten. Dabei ist allerdings zu berücksichtigen, dass diese Merkmale meist eng miteinander verknüpft auftreten, d. h. dass Menschen, die in großen Heimeinrichtungen leben, in der Regel eine stärkere geistige Behinderung aufweisen als solche, die außerhalb solcher Einrichtungen leben. Der relative Einfluss dieser Faktoren ist daher nicht eindeutig zu bestimmen.

\section{Erklärungsmodelle}

Während traditionelle psychiatrische Sichtweisen selbstverletzende Verhaltensweisen als nicht funktional bezeichneten, werden in modernen bio-psychosozialen Modellen neurobiologisch-organische Erklärungsansätze in Verbindung mit positiven und negativen so- 
zialen Verstärkungsprozessen sowie spezifischen Reizkonstellationen als Auslösebedingungen diskutiert.

Dieser Sichtweise liegen zahlreiche empirische Forschungsarbeiten zugrunde, die die funktionalen Zusammenhänge des Auftretens selbstverletzender Verhaltensweisen analysiert haben. So lässt sich bei den meisten selbstverletzenden Verhaltensweisen nachweisen, dass sie durch negative Verstärkung aufrechterhalten werden und die Funktion haben, unangenehme Anforderungen zu vermeiden, oder durch positive Verstärkung bestärkt werden und die Funktion haben, soziale Aufmerksamkeit zu erlangen. Bei einem weiteren Teil der Verhaltensweisen lassen sich automatische Verstärkungen im Sinne einer Selbststimulation durch einen bestärkenden sensorischen Reiz feststellen. Allerdings lässt sich nicht in jedem Fall eine solche Funktion eindeutig identifizieren. Unter den kritischen Auslösebedingungen ist z. B. an akute oder chronische Schmerzzustände zu denken, Schlafdefizite oder Überforderung durch Umgebungsreize, die von den Betroffenen aufgrund fehlender sprachlicher Ausdrucksmöglichkeiten nicht anders kommuniziert werden können.

Selbstverletzendes Verhalten gehört zu den charakteristischen Verhaltensmerkmalen bei einer Reihe von genetischen Syndromen, dem Lesch-Nyhan- und Cornelia-de-Lange-Syndrom, dem Rett-Syndrom, Prader-Willi-, Fragiles-X-, Cri-du-Chat- und Smith-Magenis-Syndrom (Sarimski 2014). Sie sind in vielen Fällen mit impulsiven und zwanghaften Verhaltensmerkmalen assoziiert. Das spricht für die Rolle genetischer Dispositionen als eine Bedingung für ihr Auftreten. Allerdings zeigen die Untersuchungen zu diesen Verhaltensphänotypen, dass selbstverletzende Verhaltensweisen bei Kindern, Jugendlichen und Erwachsenen mit diesen Syndromen zwar jeweils mit einer höheren Wahrscheinlichkeit auftreten als bei Menschen mit einer intellektuellen Beeinträchtigung anderer
Ursache, jedoch keinesfalls in jedem Fall und in jeder Altersgruppe mit der gleichen Häufigkeit und Ausprägung. Lediglich beim Lesch-NyhanSyndrom gehören spezifische selbstverletzende Verhaltensweisen zu den Verhaltensmerkmalen bei allen Betroffenen.

\section{Diagnostik}

Um die Symptomatik, Häufigkeit und Intensität von selbstverletzenden Verhaltensweisen einzuschätzen, eignet sich das „Inventar für Verhaltensprobleme“ (dt. Version des „Behavior Problems Inventory“, BPI-01, Sarimski \& Steinhausen 2007). Dieses erfasst neben stereotypen und aggressiven Verhaltensweisen 14 Symptome selbstverletzenden Verhaltens, die jeweils auf einer 5- bzw. 3-stufigen Skala zur Häufigkeit und Intensität eingeschätzt werden können. Die psychometrische Qualität erwies sich in internationalen Untersuchungen als gut.

Für die diagnostische Einschätzung der Zusammenhänge selbstverletzenden Verhaltens ist eine Kombination von standardisierten Fragebögen, mit denen Eltern, Pädagogen oder andere Bezugspersonen nach ihren Beobachtungen befragt werden, und systematischen Verhaltensbeobachtungen zu empfehlen. Das „Inventar zur funktionellen Erfassung selbstverletzenden Verhaltens bei Menschen mit intellektueller Beeinträchtigung" (IfES; Bienstein \& Nußbeck 2010) besteht z.B. aus 24 Items, die Situationen, in denen selbstverletzendes Verhalten auftritt, auf einer fünfstufigen Antwortskala ( $\mathrm{o}=$ nie ...4 4 immer) erfassen. Die Items sind zu folgenden fünf Subskalen zusammengefasst, die sich faktorenanalytisch bestätigen ließen: 1) situative Überforderung, 2) Erhalt eines Objekts, 3) Vermeidung von Anforderungen, 4) körperliches Unwohlsein und 5) Stimulation. Die Kennwerte für die interne Konsistenz der Subskalen, die Interrater-Reliabilität und die Test-Retest-Reliabilität sind befriedigend. 
Bei den systematischen Verhaltensbeobachtungen ist zwischen der „experimentellen Verhaltensanalyse" und der Verhaltensbeobachtung unter natürlichen Bedingungen zu unterscheiden. Bei der „experimentellen Verhaltensanalyse" wird die Häufigkeit des problematischen Verhaltens für jeweils zuvor festgelegte Zeitintervalle unter einer von mehreren Bedingungen gemessen, z. B. soziale Aufmerksamkeit des Erwachsenen kontingent auf problematisches Verhalten, unmittelbare Anforderungen an das Kind, bloße Nähe des Erwachsenen ohne aktive Interaktion oder Angebot einfachen Beschäftigungsmaterials in Abwesenheit des Erwachsenen. Variationen in der Auftretenshäufigkeit des Verhaltens in Abhängigkeit von den verschiedenen Bedingungen legen Hypothesen über funktionale Zusammenhänge nahe. Bei der Verhaltensbeobachtung unter natürlichen Bedingungen müssen vorausgehende Bedingungen (Situationsmerkmale) und Konsequenzen in einem herkömmlichen „A-B-C-Protokoll“ dokumentiert werden. Aus wiederkehrenden Mustern lassen sich ebenfalls Hypothesen über funktionale Zusammenhänge bilden.

\section{Behandlungsplanung}

Grundsätzlich belegt die Forschung, dass Behandlungsmaßnahmen, die auf einer sorgfältigen funktionalen Analyse beruhen, wesentlich erfolgreicher sind, als wenn eine solche ausführliche Diagnostik nicht durchgeführt wurde. Allerdings lassen sich nicht in jedem Fall eindeutige funktionale Zusammenhänge identifizieren. In diesen Fällen kann ein pharmakologischer Behandlungsversuch unter kontrollierten Bedingungen sinnvoll sein. Auch müssen mögliche körperliche Erkrankungen als Auftretensbedingungen berücksichtigt werden. Es ist daher zu empfehlen, die Behandlungsplanung bei selbstverletzendem Verhalten immer interdisziplinär anzulegen.

\section{Literatur}

Bienstein, P. \& Nussbeck, S. (2010): Inventar zur funktionellen Erfassung selbstverletzenden Verhaltens. Hogrefe, Göttingen

Bienstein, P. \& Rojahn, J. (2013): Selbstverletzendes Verhalten bei Menschen mit geistiger Behinderung. Hogrefe, Göttingen

Cooper, S., Smiley, E., Allan, L. et al. (2009): Adults with intellectual disabilities: prevalence, incidence and remission of self-injurious behavior, and related factors. Journal of Intellectual Disability Research, 53, 200-216.

Rojahn, J., Borthwick-Duffy, S. \& Jacobson, J. (1993): The association between psychiatric diagnoses and severe behavior problems in mental retardation. Annals of Clinical Psychiatry, 5, $163-170$.

Sarimski, K. (2014): Entwicklungspsychologie genetischer Syndrome. 4. erweiterte und überarbeitete Auflage. Hogrefe, Göttingen

Sarimski, K. \& Steinhausen, H.-C. (2007): Geistige Behinderung und schwere Entwicklungsstörung. KIDS-2. Hogrefe, Göttingen 